Revista Mexicana de Análisis de la Condueta
Revista Mexicana de Análisis de la Conducta

ISSN: 0185-4534

editora@rmac-mx.org

Sociedad Mexicana de Análisis de la Conducta

México

GONZÁLEZ-BECERRA, VICTOR HUGO; ORTIZ RUEDA, GERARDO ALFONSO EFECTOS DE LA RETROALIMENTACIÓN Y EL TIPO DE DESCRIPCIÓN DE CONTINGENCIAS SOBRE LA CONDUCTA DE DISCRIMINACIÓN CONDICIONAL EN UNA TAREA DE IGUALACIÓN DE LA MUESTRA

Revista Mexicana de Análisis de la Conducta, vol. 40, núm. 1, junio, 2014, pp. 51-80 Sociedad Mexicana de Análisis de la Conducta Distrito Federal, México

Disponible en: http://www.redalyc.org/articulo.oa?id=59335810003

Cómo citar el artículo

- Número completo

- Más información del artículo

- Página de la revista en redalyc.org

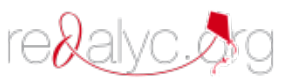

Sistema de Información Científica

Red de Revistas Científicas de América Latina, el Caribe, España y Portugal Proyecto académico sin fines de lucro, desarrollado bajo la iniciativa de acceso abierto 


\title{
EFECTOS DE LA RETROALIMENTACIÓN Y EL TIPO DE DESCRIPCIÓN DE CONTINGENCIAS SOBRE LA CONDUCTA DE DISCRIMINACIÓN CONDICIONAL EN UNA TAREA DE IGUALACIÓN DE LA MUESTRA
}

\author{
EFFECTS OF FEEDBACK AND THE TYPE OF CONTINGENCY \\ DESCRIPTION ON CONDITIONAL DISCRIMINATION IN A \\ MATCH-TO-SAMPLE TASK
}

\section{VICTOR HUGO GONZÁLEZ-BECERRA ${ }^{(1)}$ GERARDO ALFONSO ORTIZ RUEDA ${ }^{(2)}$}

\author{
UNIVERSIDAD DE GUADALAJARA, CENTRO UNIVERSITARIO DE LOS \\ VALLES / CENTRO DE ESTUDIOS E INVESTIGACIONES EN \\ COMPORTAMIENTO
}

\begin{abstract}
Resumen
Se determinaron los efectos de la retroalimentación y el tipo de descripción de contingencias sobre la adquisición y transferencia de una conducta de discriminación condicional en una tarea de igualación de la muestra de primer orden (TIM-1). En el Experimento 1 se encontró que la mayoría de los participantes que recibieron descripciones específicas y pertinentes (EP) y descripciones correctas e incorrectas $(\mathrm{Cl})$ obtuvieron un porcentaje de respuestas correctas cercano al $100 \%$ en el entrenamiento. El porcentaje de respuestas correctas disminuyó en las pruebas de

(1) Doctorante en Ciencia del Comportamiento, opción Análisis de la Conducta, en el Centro de Estudios e Investigaciones en Comportamiento (CEIC) de la Universidad de Guadalajara. El desarrollo de esta investigación fue gracias al apoyo recibido por el CONACYT mediante la beca \#334736.

(2) Profesor-investigador del CEIC de la Universidad de Guadalajara.

Ambos autores trabajaron en la misma proporción en la elaboración de la presente investigación; la correspondencia relacionada con este trabajo puede enviarse a Centro de Estudios e Investigaciones en Comportamiento, Francisco de Quevedo No. 180, Col. Arcos Vallarta, Guadalajara, Jalisco, México, 44130; o al e-mail: victor.hugo.glez@gmail.com.
\end{abstract}


transferencia, sin importar la descripción de contingencias recibida. Este resultado posiblemente se debió a la falta de una señal agregada que especificara la respuesta esperada del participante a través de las sesiones. El Experimento 2 fue una réplica del Experimento 1, excepto porque se agregó un aviso de la sesión experimental enfrentada (i.e., preprueba, entrenamiento, posprueba, pruebas de transferencia) al inicio de cada sesión. Añadir la señal resultó en un porcentaje alto de respuestas correctas durante todas las sesiones. Las descripciones EP y $\mathrm{Cl}$ favorecieron la adquisición y transferencia de una conducta de discriminación condicional. Se discutieron los efectos teóricos y metodológicos relacionados al estudio de la conducta sustitutiva referencial.

Palabras clave: descripción de contingencias, transferencia, igualación de la muestra, conducta sustitutiva referencial

\begin{abstract}
The effects of feedback and the type of the contingency description on the acquisition and transfer of conditional discrimination in a first order matching-to-sample task were assessed. Experiment 1 showed that participants who received specific and relevant descriptions (SR) and correct and incorrect descriptions (Cl) obtained almost $100 \%$ of correct responses during training. The percentage of correct responses decreased abruptly during transfer tests, regardless of the description of contingencies received. Experiment 2 was similar to Experiment 1, except that a signal that specified which experimental condition was in effect was added during each session (i.e., pre-test, training, post-test, transfer tests). The addition of the signal resulted in high percentages of correct responses during all the sessions. The SR and $\mathrm{Cl}$ descriptions lead to the acquisition and transfer of matching. Theoretical and methodological aspects of the study of referential substitution behavior were discussed.

Keywords: contingency description, transfer, matching to sample, referential substitution.

La teoría interconductual ribesiana (TIR) (Ribes \& López, 1985; Ribes, 1992, 2004,2006 , 2007) propone que el comportamiento psicológico se puede clasificar en cinco categorías denominadas funciones, a saber: contextual, suplementaria, selectora, sustitutiva referencial y sustitutiva no referencial. El término función hace referencia al sistema de interrelaciones que se establece en la interacción de un organismo o individuo con objetos, eventos, organismos o individuos. Cada una de las
\end{abstract}

1 Se denominará Teoría Interconductual Ribesiana (TIR) a la propuesta realizada por E. Ribes para distinguirla de las propuestas de J. R. Kantor y J. Roca. 
funciones se va haciendo progresivamente más compleja en cuanto a la organización de las interrelaciones que se establecen en la interacción, caracterizándose, entre otras cosas, por la forma en la que la conducta se ajusta a las circunstancias.

La función menos compleja es la función contextual porque el criterio de ajuste conductual solo implica la diferencialidad en la reacción del organismo a un estímulo interrelacionado a una relación estímulo-respuesta establecida antes de la interacción. El segundo grado de complejidad conductual lo ocupa la función suplementaria debido a que el criterio de ajuste conductual de este sistema interactivo implica la efectividad en la acción del organismo para suplementar una relación entre estímulos, comportamiento que requiere de la "reacción diferencial" a los estímulos que se suplementan. La función selectora se clasifica en el tercer grado de complejidad conductual porque para que la respuesta del organismo/individuo sea "efectiva" tiene que "reaccionar diferencialmente" a un estímulo que se vuelve la condición para que su respuesta suplemente una relación entre estímulos; en este sentido se dice que el criterio de ajuste conductual en esta función es la precisión. En el cuarto nivel de complejidad conductual se encuentra la función sustitutiva referencial, cuyo criterio de ajuste es la congruencia de la respuesta de un individuo a los criterios de respuesta a los que responde otro individuo. Cabe señalar que este tipo de interacción sólo se considera sustitutivo referencial cuando los criterios de "precisión" en la respuesta del individuo a un mismo objeto o evento son sustituidos por la acción de otro individuo. En este sentido la función sustitutiva referencial implica dos "respuestas precisas" relacionadas a dos situaciones distintas, pero vinculadas a un mismo objeto o evento, donde la "respuesta precisa" relacionada a la situación sustituyente es la que se considera congruente. Por último, el quinto grado de complejidad conductual corresponde a la función sustitutiva no referencial y el criterio de ajuste conductual es la coherencia. Se considera que este es el tipo de comportamiento más complejo porque requiere que la respuesta de un individuo interrelacione dos situaciones distintas para crear una nueva situación interactiva de manera coherente. Este tipo de interacción sólo se posible en individuos que "responden congruentemente" con relación a dos situaciones vinculadas a un mismo objeto.

Es necesario precisar que las situaciones no deben de confundirse con el lugar físico en el que ocurre la interacción, ni con los objetos físicos con los que se interactúa, más bien deben de identificarse en las circunstancias o condiciones que delimitan la interacción (e. g., Ribes, 1990a; Torres, Ortiz, Rangel, \& González, 2010). En el caso de las situaciones que se mencionan en las funciones sustitutivas las circunstancias son de naturaleza convencional, es decir, que se articulan a partir del acuerdo implícito o explícito de lo que se puede y lo que no se puede hacer en la práctica entre individuos.

Tomando en cuenta la participación de la situación o situaciones en la organización de las interrelaciones de cada función se proponen tres tipos de categorías 
conductuales, a saber: intrasituacionales, extrasituacionales y transituacionales. Las tres funciones menos complejas (contextual, suplementaria y selectora) se denominan intrasituacionales porque se organizan a partir de una situación particular. La circunstancia o situación en la que se establecen las funciones contextual y suplementaria ligan la respuesta del organismo a las propiedades absolutas de los objetos con los que se interactúa, un cambio en sus propiedades imposibilita la interacción. En cambio, el nivel de desligamiento de la respuesta evoluciona en la función selectora, pues se desliga de las propiedades absolutas de los objetos y se liga a sus propiedades relativas, las cuales cambian si el elemento que condiciona la respuesta que suplementa la relación entre los estímulos relacionados a la "respuesta efectiva" cambia. Pero aunque en la función selectora las relaciones entre algunos de sus elementos cambian de un momento a otro la situación sigue siendo la misma porque la configuración de relaciones posibles se mantiene constante en la interacción. Por otra parte la función sustitutiva referencial también se denomina función extrasituacional porque la situación que delimita la forma en la que un individuo responde a un objeto se sustituye por una nueva situación, una "situación extra". Al respecto Ribes (2012) señaló: "La sustitución extrasituacional ocurre sólo cuando los cambios de comportamiento y sus resultados no constituyen parte de las relaciones condicionales prescritas "usualmente" en la situación. (p. 23)". En otras palabras la sustitución extrasituacional implica la sustitución de la configuración de las relaciones posibles que delimitan las formas en las que el individuo puede interactuar con un mismo objeto o evento, donde el individuo desliga su comportamiento de los criterios relacionados a una situación para ligarlos a los criterios de otra situación. En el caso de la función sustitutiva no referencial la respuesta del individuo no se delimita por ninguna situación particular, pues es el individuo quien crea una nueva situación a partir de la interrelación de cuando menos dos situaciones; por eso se le denomina sustitución transituacional (e. g., Ribes, 1990b).

Inicialmente Ribes (1990b) propuso que para evaluar el comportamiento intrasituacional, extrasituacional y transituacional sólo era necesario hacer algunas variaciones al desarrollo original de la Tarea de Igualación de la Muestra (TIM) (Skinner, 1950; Cumming \& Berryman, 1961). El procedimiento base de la TIM consiste en relacionar un Estímulo Muestra (EM) a uno de varios Estímulos Comparativos (Eco's) con base en un criterio de respuesta (i.e., semejanza, identidad, diferencia), lo cual permite evaluar el comportamiento instrasituacional del participante. Pero para evaluar el comportamiento extrasituacional y transituacional se propuso la creación de sesiones en las que los estímulos utilizados variaran respecto de los utilizados en el entrenamiento. A estas sesiones se les conoce tradicionalmente como pruebas de transferencia porque se espera que el participante "transfiera" lo que aprendió en el entrenamiento a las sesiones en las que se varían los estímulos o sus relaciones. En este sentido se propuso 
que si el participante era capaz de responder acertadamente al cambio de criterio de relación entre el EM y uno de los Ecós (i.e., de semejanza a diferencia) sería evidencia de comportamiento sustitutivo referencial, es decir, extrasituacional. Con relación a dicha prueba de transferencia Ribes (1990b) mencionó:

“...se mantienen los mismos estímulos y modalidades que en la situación original, pero se modifica la relación... En esta condición el niño debe de responder de forma sustitutiva referencial, es decir, debe de responder a los mismos estímulos y modalidad con base en un reconocimiento de un cambio de relación, externo a la situación. Su respuesta lingüística a la nueva relación debe sustituir las relaciones de identidad por las de diferencia" (p. 218).

Por último, Ribes (1990b) propuso que cuando el participante respondía acertadamente a una prueba de transferencia en la que variaban la relación, la modalidad (modo de presentación de los estímulos, por ejemplo rotación o tamaño) y la dimensión de los objetos utilizados como estímulos (i.e., geométrica, aritmética) su comportamiento era sustitutivo no referencial, o sea, transituacional. En resumen, la propuesta se reduce a identificar el comportamiento instrasituacional con respuestas de discriminación condicional correctas a estímulos que no varían en una TIM. Mientras que el comportamiento extrasituacional se identifica con respuestas de discriminación condicional correctas en sesiones en las que el criterio de relación cambio respecto del entrenamiento y el comportamiento transituacional con respuestas correctas pese a la variación en el criterio de relación, la modalidad y la dimensión de los estímulos respecto a los utilizados en el entrenamiento.

Una gran cantidad de investigaciones se han realizado con base en la propuesta de Ribes (1990b) para la evaluación del comportamiento intra, extra y transituacional (e. g., Pérez \& Flores, 2007; Ribes, Ontiveros, Torres, Calderón, Carvajal, Martínez, \& Vargas, 2005; Ribes, Moreno, \& Martínez, 1995; Serrano, García, \& López, 2006; Serrano, García, \& López, 2008; Serrano, García, \& López, 2009; Serrano, García, \& López, 2010; Villanueva, Flores, \& Mateos, 2008). Los resultados de las investigaciones no son concluyentes debido a que se encuentran diferencias en la ejecución de los participantes que fueron expuestos a las mismas condiciones (Peña-Correal, Ordóñez, Fonseca, J., \& Fonseca, C. 2012). Sin embargo, hay algunos datos consistentes en los resultados respecto a la manipulación de la retroalimentación de la ejecución correcta o incorrecta de los participantes y del tipo de descripciones de contingencias (de relaciones de dependencia entre estímulos y respuestas) que reciben previo o posterior a su ejecución.

Uno de los efectos más contundentes de la retroalimentación continua sobre la ejecución de los participantes que interactúan en TIM es la reducción en el número de ensayos necesarios para adquirir y mantener una conducta de discriminación condicional respecto de los participantes que reciben retroalimentación demorada, 
es decir, que reciben información de su ejecución correcta o incorrecta al final de la sesión (Martínez, Ortiz, \& González, 2007; Ortiz, González, Rosas, \& Alcaráz, 2006; Varela \& Quintana, 1995). Sin embargo, también se ha encontrado que la retroalimentación continua no favorece el mantenimiento de la ejecución correcta del entrenamiento en las pruebas de transferencia; en cambio se ha encontrado que la retroalimentación acumulada si favorece la transferencia (Moreno, Ribes, \& Martínez, 1994; Ribes \& Martínez, 1990; Serrano, García, \& López, 2009).

Otro tipo de variable que se manipula frecuentemente para evaluar su efecto sobre la adquisición y transferencia de una conducta de discriminación condicional es la descripción de contingencias que el participante recibe previo, durante o posterior a su ejecución. Las descripciones de contingencias se presentan al participante con la finalidad de fungir como instrucciones, es decir, como eventos que promueven el ajuste conductual a un tipo de criterio (e. g., Ortiz, González, \& Rosas, 2008). Sin embargo, no siempre se encuentra correspondencia entre la conducta señalada en las descripciones de contingencias y la conducta del participante que las recibió (Martínez et al., 2007; Ribes \& Martínez, 1990). Algunos de los factores que modulan la correspondencia entre la conducta señalada en las descripciones de contingencias y la conducta del participante que las recibe son: a) la precisión de la descripción, b) las propiedades descritas de la respuesta y estímulos y c) el ejemplar o ejemplares descritos.

De acuerdo con Ortiz et al. (2008) el tipo de descripción de contingencias con mayor grado de precisión es aquella en la que se describen todos los elementos relevantes para que la respuesta del participante que las recibe sea correcta; a tal tipo de descripción de contingencias le califica como Específica-Pertinente. El siguiente grado de precisión es para aquellas descripciones en las que sólo se mencionan algunos de los elementos relevantes, su calificativo Genéricas-Pertinentes. Cuando lo descrito no corresponde a la conducta que se considera correcta en una situación experimental, pero se mencionan algunos de los elementos que participan en la interacción, las descripciones de contingencias se califican como No Pertinentes, ya sea de manera Específica o Genérica. Por último, los autores califican como Irrelevantes a las descripciones de contingencias que nada tienen que ver con la conducta esperada en una situación experimental ni con los elementos que participan en ella. Respecto a la anterior clasificación de las descripciones de contingencias se han realizado algunas investigaciones en las que se estudiaron los efectos de la variación en la precisión de las descripciones sobre la conducta de discriminación condicional (e. g., Cruz \& Ortiz, 2011; Guerrero-Radillo \& Ortiz, 2007, 2009; Ortiz, 2010; Ortiz \& González, 2010; Ortiz, González, Rosas, \& Alcaraz, 2006). Los resultados encontrados sugieren que entre más precisas son las descripciones de contingencias más rápido se adquiere y mantiene la conducta de discriminación condicional en TIM. 
Con relación a las propiedades que se señalan de los estímulos y las respuestas involucradas en un tipo de interacción las descripciones de contingencias se pueden clasificar en tres tipos: 1) instanciales, caracterizadas por señalar la instancia u objeto particular con el que se interactúa; 2) modales, denominadas así por señalar el modo en el que se presentan las instancias con las que se interactúa (i.e., color, forma, tamaño, rotación, ; y c) referentes al criterio de relación entre las instancias con las que se interactúa (i.e., semejanza, diferencia, identidad). Para algunos autores las descripciones de contingencias instanciales promueven el comportamiento intrasituacional, las modales el extrasituacional y las relacionales el extradimensional (e. g., Carpio, Pacheco, Carranza, \& Canales, 2003; Irigoyen, Carpio, Jimenéz, Silva, \& Acuña, 2002; Serrano, García, \& López, 2006; Serrano, García, \& López, 2008; Villanueva, Mateos, \& Flores, 2008). En la mayoría de las investigaciones en las que se han manipulado las descripciones de contingencias instanciales, modales y relacionales para evaluar su efecto sobre la conducta de discriminación condicional se ha encontrado que las de tipo relacional promueven los porcentajes de respuesta más altos en el entrenamiento y en las pruebas de transferencia (e. g., González-Becerra \& Ortiz, 2014; Ribes, Moreno, \& Martínez, 1995; Ribes, Moreno, \& Martínez, 1998; Serrano, García, \& López, 2006; Serrano, García, \& López, 2008; Villanueva, Mateos, \& Flores, 2008). Pero, también se pueden encontrar investigaciones en las que los porcentajes de respuesta más altos en las pruebas de transferencia lo obtienen los participantes que reciben descripciones modales (e. g., Serrano et al., 2010).

Una cualidad adicional que se puede manipular de las descripciones de contingencias es el ejemplar conductual que se describe al participante, el cual generalmente se enfoca en la descripción de la respuesta pertinente a la tarea experimental, quedando sin describir aquellas conductas no pertinentes. Al respecto Engelmann y Carnine (1991) señalan que cuando a un individuo se le presentan ejemplares pertinentes y no pertinentes de un tipo de respuesta tiene más probabilidades de mantener y generalizar la respuesta aprendida que cuando sólo se le presentan ejemplos pertinentes (Ribes, 2001). Una investigación que apoya el anterior supuesto es la de Serrano, García y López (2009). Los investigadores expusieron a tres grupos de participantes a diferentes tipos de retroalimentación de su respuesta en una TIM, a saber: 1) retroalimentación de respuestas correctas e incorrectas, 2) retroalimentación de respuestas correctas y 3) retroalimentación de respuestas incorrectas. Se encontró que los participantes que recibieron retroalimentación de sus respuestas correctas e incorrectas mantuvieron porcentajes de aciertos cercanos al $100 \%$ en el entrenamiento y en las pruebas de transferencia, mostrando mejores ejecuciones que aquellos participantes que sólo recibieron retroalimentación de sus respuestas correctas o incorrectas.

Tomando en cuenta lo comentado en párrafo inmediato anterior Ortiz \& González (2010) realizaron una investigación con el objetivo de evaluar si la descripción de con- 
tingencias en la que se presentan ejemplos de repuesta correctos e incorrectos tenía un efecto similar a los de la retroalimentación de respuestas correctas e incorrectas. Para tales propósitos en dicha investigación tres grupos de participantes recibieron descripciones de contingencias con un ejemplo de conducta pertinente a la TIM y otros tres grupos recibieron descripciones de contingencia con varios ejemplos de respuestas correctas e incorrectas. Los resultados mostraron que ambas descripciones de contingencias promovieron porcentajes de acierto cercanos al 100\% en las sesiones de entrenamiento, pero no fue así en las pruebas de transferencia, pues en esas sesiones los participantes obtuvieron porcentajes de respuestas de aciertos cercanos a cero. Los autores sugirieron que posiblemente variables como las autoinstrucciones (Cerutti, 1989) pudieron influir en los resultados, pues al menos los bajos porcentajes de aciertos en las sesiones de transferencia de los participantes que recibieron descripciones de contingencias con un solo ejemplo de repuesta correcta no corresponden a los altos porcentajes de acierto en la mayoría de las sesiones de transferencia de participantes que recibieron el mismo tipo de descripción de contingencias en otras investigaciones (e. g., Serrano, García, \& López, 2006; Serrano, García, \& López, 2009).

El objetivo de la presente investigación es realizar un experimento como el de Ortiz y González (2010) en cuanto al propósito de evaluar si señalar ejemplos de respuesta correcta e incorrecta en la descripción de contingencias promueve la adquisición y transferencia de una conducta de discriminación condicional en una TIM. La única diferencia de la presente investigación con su precedente es que se excluirán del análisis los grupos de participantes que recibieron retroalimentación continua porque se ha encontrado en otras investigaciones que dicha variable no favorece la transferencia de la conducta aprendida en el entrenamiento (Ribes \& Martínez, 1990). Por último, con relación a la posible influencia de las autoinstrucciones (Cerutti, 1989; Ribes, 2000) en los resultados no se desarrolló ningún cambio en la tarea, sólo se compararán los resultados de ambas investigaciones.

\section{Experimento 1}

Con base en un diseño factorial $2 \times 2$, en el que se combinaron dos tipos de descripciones de contingencias Específicas-Pertinentes (EP) y Correctas e Incorrectas $(\mathrm{Cl})$ con dos densidades de la retroalimentación (acumulada y ausente), se evaluó el efecto de dichas variables sobre la adquisición y transferencia de una repuesta de discriminación condicional. El propósito del estudio fue evaluar si las descripciones de contingencias con ejemplos de respuesta correcta e incorrecta promueven respuestas correctas en el entrenamiento y sesiones de transferencia como cuando se manipula la exposición directa con la retroalimentación de respuestas correctas e incorrectas (e. g., Serrano, García, \& López, 2009). Los grupos de participantes que recibieron 
descripciones Específicas-Pertinentes (EP) con un solo ejemplo de respuesta correcta fungieron como controles de la ejecución de los que recibieron descripciones Incorrectas-Correctas (IC). Por último, la densidad de la retroalimentación se manipuló con la finalidad de evaluar los efectos de las descripciones de contingencias con y sin retroalimentación.

\section{Método}

Participantes. Participaron 32 estudiantes universitarios con edades entre 18 y 23 años, sin experiencia en la tarea experimental, los cuales se asignaron al azar a los grupos experimentales. El contacto con los participantes fue a través de sus profesores, quienes les dieron puntos extras en su asignatura por la participación en el experimento.

Aparatos y materiales. Todas las sesiones experimentales se llevaron a cabo en cuatro cubículos ubicados dentro de las instalaciones del Centro de Estudios e Investigaciones en Comportamiento de la Universidad de Guadalajara. Los cubículos tenían dimensiones aproximadas de 3 metros de largo por 3 metros de ancho, iluminación artificial y contenían un mueble con dos sillas y una computadora personal con ambiente Windows XP. La programación de los estímulos, la aplicación de la tarea experimental y el registro de las respuestas de los participantes se llevó a cabo mediante el programa Toolbook Instructor I/®.

Diseño. Se utilizó un diseño factorial $2 \times 2$ (tipo de descripción de contingencias $x$ densidad de retroalimentación), lo cual dio lugar a cuatro grupos. Las variables que se combinaron fueron la retroalimentación acumulada y la retroalimentación ausente con las descripciones específicas y pertinentes (EP) con un solo ejemplo de respuesta correcta y las descripciones de respuestas correctas e incorrectas $(\mathrm{Cl})$. Los grupos que resultaron de dicha combinación fueron el Grupo EP-Acumulada (Grupo 1), el Grupo EP-Ausente (Grupo 2), el Grupo Cl-Acumulada (Grupo 3) y el Grupo ClAusente (Grupo 4). Todos los grupos enfrentaron una sesión de preprueba, posprueba, así como de pruebas de transferencia extrainstancial, extramodal, extrarelacional y extradimensional (de acuerdo a la matriz de transferencias propuesta por Varela y Quintana, 1995); mientras que en el entrenamiento podían enfrentar de tres o cuatro sesiones (ver Tabla 1).

Tarea experimental. Como tarea experimental se utilizó un procedimiento de igualación de la muestra de primer orden (TIM-1). Cada ensayo consistió en la presentación de un estímulo muestra (EM) colocado al centro de la pantalla y tres estímulos comparativos (Eco's) alineados horizontalmente en la parte inferior de la pantalla. Cada arreglo de estímulos presentaba, respecto al EM, un ECo idéntico, uno semejante y otro diferente. La tarea de cada participante consistió en elegir de entre 


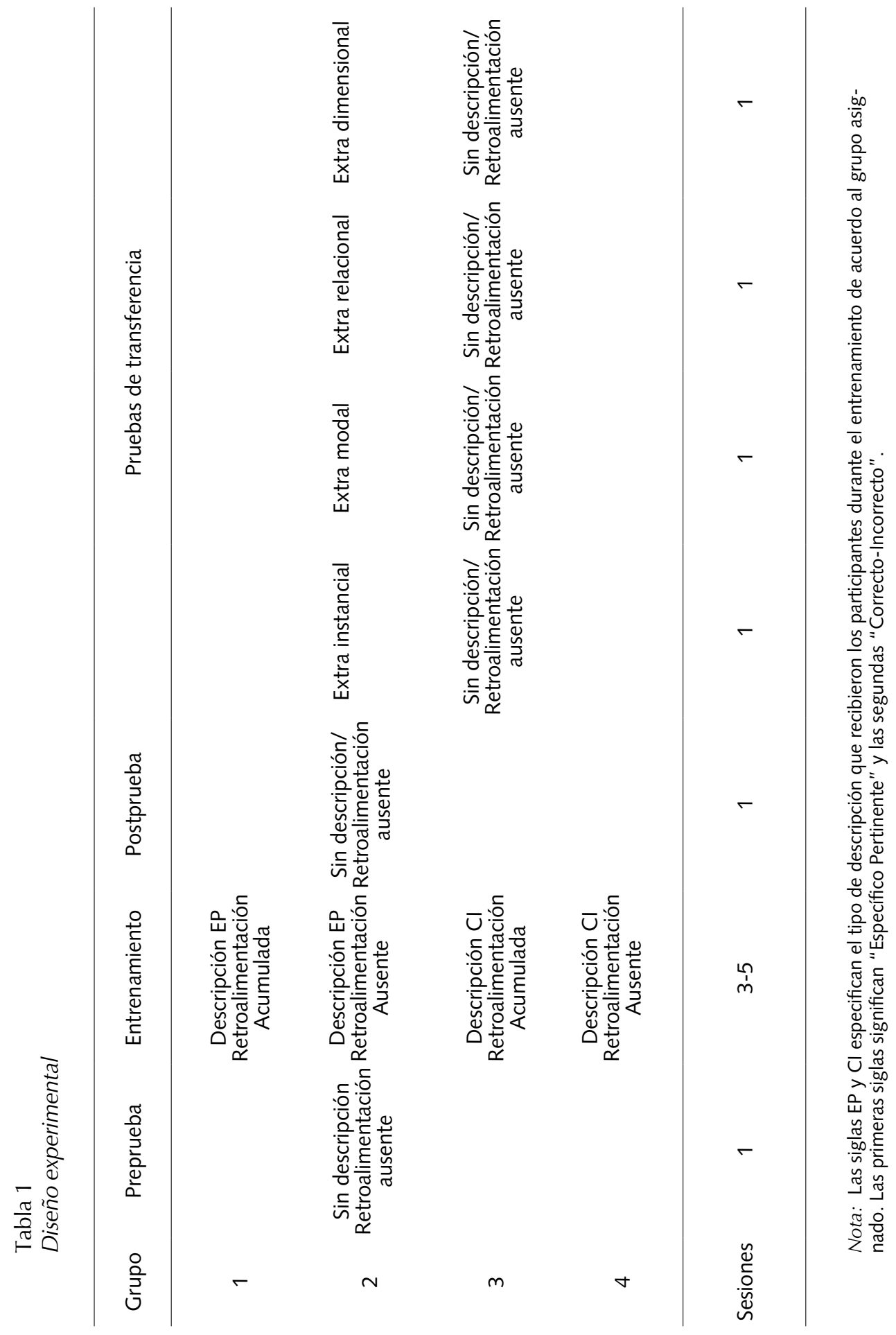


los tres Eco's, aquel que guardaba la relación de semejanza con el EM, excepto en las pruebas de transferencia extrarelacional y extradimensional, en las cuales el criterio de relación cambiaba a identidad o diferencia.

Las figuras empleadas en las sesiones de preprueba, entrenamiento y posprueba fueron cuadros, triángulos, rectángulos y círculos de color blanco, rojo, verde y amarillo. En la prueba de transferencia extrainstancial se utilizaron pentágonos, hexágonos, rombos y trapecios de color naranja, azul, rosa y morado; en la prueba de transferencia extramodal se utilizaron las mismas figuras que en la prueba anterior, cambiando los tamaños de las figuras (chicas y grandes) manteniendo un mismo color para todos los estímulos (gris). En la prueba de transferencia extrarelacional se utilizaron las mismas figuras que enla anterior prueba, solo cambió el criterio de igualación a diferencia o identidad. Por último, en la transferencia extradimensional los estímulos fueron números de dos cifras, donde los Eco's se podían igualar ya sea por decenas o unidades respecto al EM y el criterio de igualación que se consideró correcto fue el de diferencia o identidad, dependiendo del criterio seleccionado por el participante en la prueba extrarelacional (véase Tabla 2). El arreglo y el tipo de estímulos utilizados en cada sesión fue el mismo para todos los grupos experimentales (véase Figura 1).

\begin{tabular}{|l|l|l|}
\hline Sesión Experimental & Estímulos utilizados \\
Preprueba, entrenamiento &
\end{tabular}

Figura 1. Estímulos utilizados en las sesiones experimentales. 


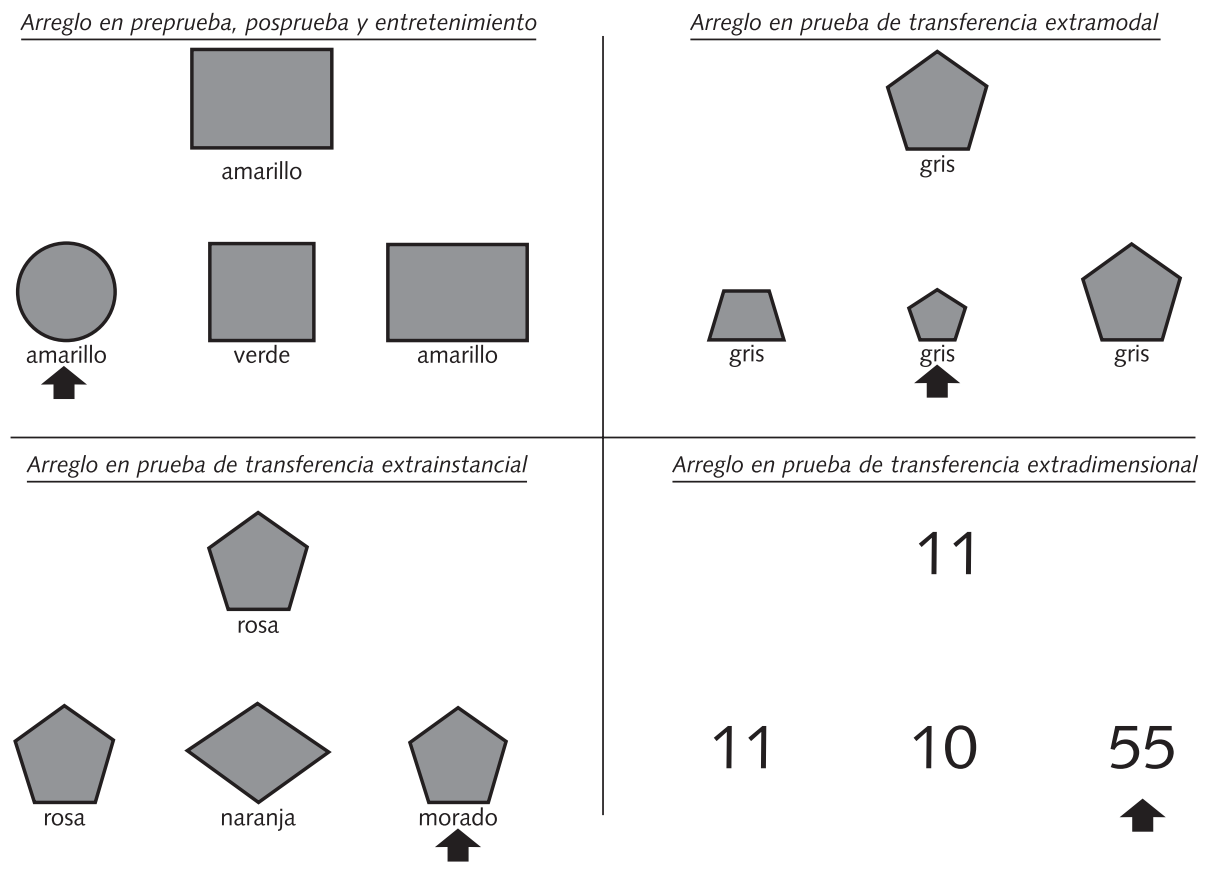

Figura 2. Arreglos de estímulos en las sesiones experimentales. En cada ejemplo de arreglo hay un estímulo comparativo diferente, idéntico y semejante. Las flechas indican el estímulo correcto en cada ejemplo (en todos ejemplos que contienen figuras geométricas se señala la figura semejante, mientras que la que contiene números se señala la diferente).

Procedimiento. Al inicio del estudio a cada participante se le pidió que se sentara frente al monitor y oralmente se le indicó que se trataba de un estudio sobre aprendizaje, agradeciendo su participación en el mismo. Además, se les mencionó que no se traba de una prueba de inteligencia o un test proyectivo, que lo anterior era toda la información que el asistente podría brindarles y que al final del experimento se le explicarían los propósitos de dicho trabajo y los resultados de su ejecución, en caso de que así lo desearan.

Al comenzar cada sesión se presentó en la pantalla del monitor una diapositiva de bienvenida al experimento. En la siguiente diapositiva se encontraban descripciones que variaron dependiendo del grupo y de la fase experimental en la que se encontró cada participante. Posteriormente se presentaban, en una serie de diapositivas, los arreglos de estímulos a los que tenían que responder los participantes. Al final de la sesión se presentaba en una diapositiva la indicación de que la sesión había termina- 
do y que era necesario llamar al encargado del experimento. Cuando esto ocurría el encargado guardaba los datos, cargaba en el computador una nueva sesión y dejaba solo al participante en el cubículo para que continuara con el experimento. Todos los participantes enfrentaron inicialmente una preprueba, en la cual no se retroalimentaba la ejecución. En seguida enfrentaban la fase de entrenamiento en la que la mitad de los participantes recibieron al final de cada sesión retroalimentación de la cantidad de aciertos obtenidos (retroalimentación-acumulada), mientras que la otra mitad no recibió dicha retroalimentación (retroalimentación-ausente). Además, en combinación con la retroalimentación una mitad recibió descripciones específicas y pertinentes (EP) de la Situación de Estímulo (SE) (i.e., los elementos del ambiente relevantes en la interacción), la respuesta efectiva en dicha situación de estímulo (R) y las consecuencias derivadas de ello (C) (véase Ortiz, González, \& Rosas, 2008) y, la otra mitad recibió descripciones a manera de ejemplos correctos e incorrectos $(\mathrm{Cl})$ basados en la propuesta de generalización del conocimiento de Engelmann y Carnine (1991) (véase Anexo 1).

Todas las sesiones experimentales constaron de 36 ensayos y no tenían límite temporal de ejecución. La primera sesión enfrentada era la preprueba. Posteriormente la fase de entrenamiento constaba de un mínimo de tres sesiones y un máximo de cuatro. Cuando los participantes obtenían un porcentaje de aciertos mayor al $90 \%$ en tres sesiones consecutivas pasaban a la posprueba, de lo contrario enfrenta ban una cuarta sesión de entrenamiento. Las sesiones de preprueba y posprueba no eran retroalimentadas ni tenían descripciones. Por último, los participantes enfrentaban una fase de pruebas de transferencia en las que no se retroalimentaba su ejecución, sólo al inicio de la prueba de transferencia extrarelacional se presentaba un aviso que decía: "cambia el criterio de relación".

\section{Resultados}

En la Figura 2 se muestra en cada gráfica el porcentaje de aciertos de los participantes del Experimento 1. Las graficas están alineadas en cuatro columnas que, de izquierda a derecha, ubican las ejecuciones de los participantes del Grupo 1 (EPacumulada), Grupo 2 (EP-ausente), Grupo 3 (Cl-acumulada) y Grupo 4 (Cl-ausente). En el eje de las abscisas de cada gráfica se encuentran las sesiones experimentales y en el eje de las ordenadas el porcentaje de aciertos obtenidos. Con círculos en color negro se representa el porcentaje de respuestas correctas en la preprueba $(\mathrm{Pr})$ y posprueba (Po). De manera similar el porcentaje de respuestas correctas de los participantes en el entrenamiento (e) y en las pruebas de transferencia se representó con círculos en color negro, pero adicionalmente se unieron con líneas rectas en color negro (véase Figura 3). 


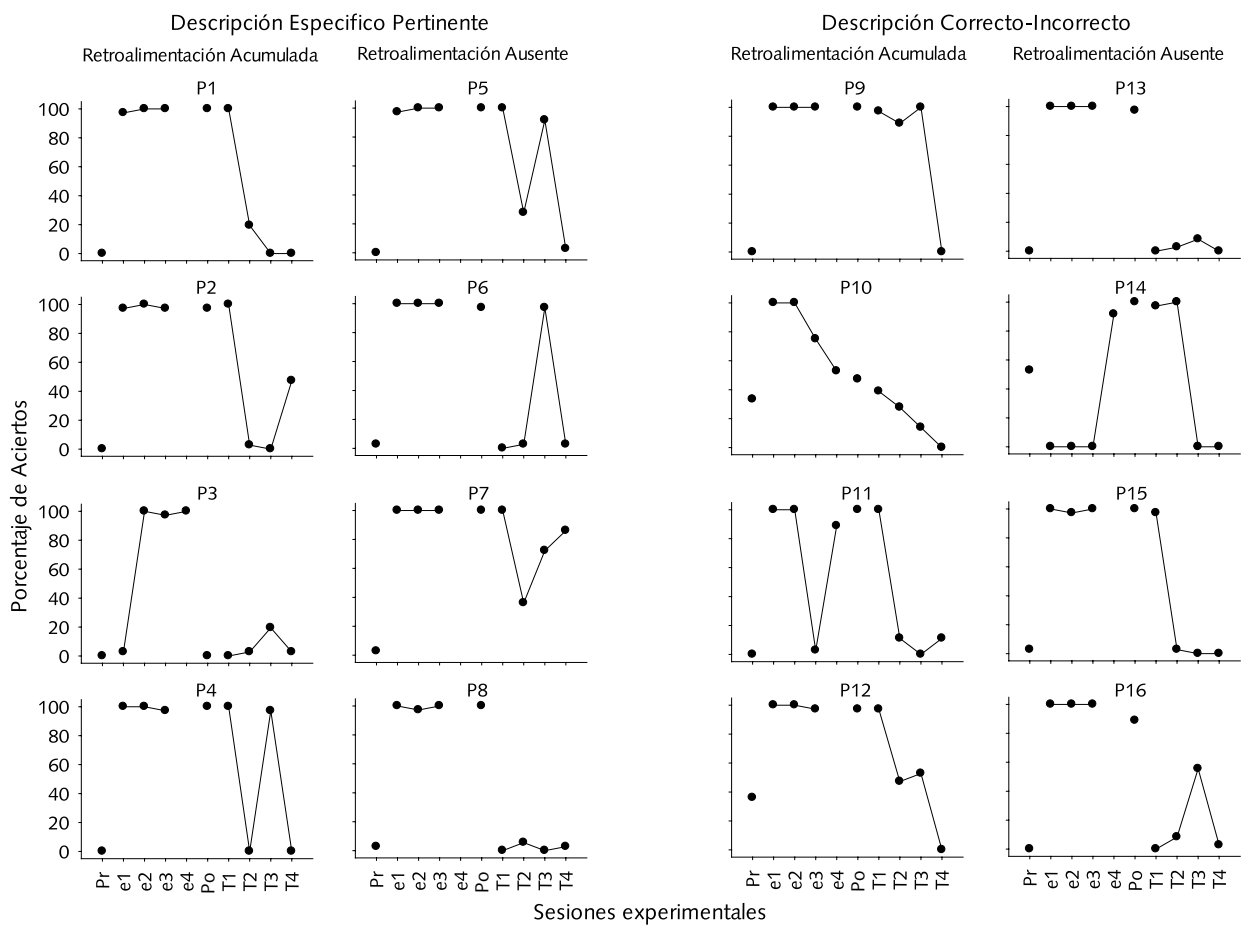

Figura 3. Porcentajes de aciertos obtenidos por los participantes del Experimento 1. Las abreviaciones representan sesiones experimentales y variables independientes, a saber: $\mathrm{Pr}=$ preprueba, $\mathrm{e}=$ entrenamiento, $\mathrm{Po}=$ posprueba, $\mathrm{T} 1$ = prueba de transferencia extrainstancial, $\mathrm{T} 2$ = prueba de transferencia extra modal, $\mathrm{T} 3=$ prueba de transferencia extrarelacional y $\mathrm{T} 4=$ prueba de transferencia extradimensional.

Con relación a las ejecuciones de los participantes en las sesiones de entrenamiento, se puede ver que casi la totalidad de los participantes que recibieron descripciones EP (con excepción de P3) obtuvieron porcentajes cercanos al 100\% de aciertos en tres sesiones consecutivas. En cambio dos de los participantes que recibieron descripciones $\mathrm{Cl}$ (P11 y $\mathrm{P} 14$ ) requirieron más de tres sesiones para lograr un porcentaje de aciertos cercano al $100 \%$ en la última sesión de entrenamiento, mientras que otro participante no logró superar dicho porcentaje de aciertos en la última sesión (P10). A grandes rasgos los resultados muestran que las descripciones EP son mejores que las $\mathrm{Cl}$ en cuanto a la adquisición de la conducta de discriminación condicional en el entrenamiento.

En la posprueba se encontró que casi todos los participantes obtuvieron porcentajes de aciertos cercanos al $100 \%$ cuando obtuvieron un porcentaje de aciertos similar en 
la última sesión del entrenamiento (con excepción de P3 que no obtuvo ningún acierto en la posprueba), independientemente del tipo de retroalimentación o descripción recibida. Sin embargo, los porcentajes de respuestas correctas cercanos al $100 \%$ no se mantuvieron del paso de la posprueba a la prueba de transferencia extrainstancial (T1) en todos los participantes, ya que cuatro de ellos (P6, P7, P13 y P16) no obtuvieron ningún acierto en dicha prueba de transferencia pese a haber obtenido casi el $100 \%$ de los aciertos en la posprueba que le precedía. De los participantes que lograron porcentajes de aciertos cercanos al 100\% de los aciertos en T1 (P1, P2, P4, P5, P6, P9, P11, P12, P14 y P15) sólo dos mantuvieron el mismo tipo de ejecución en la prueba de transferencia extramodal (T2) (P9 y P14), quienes, por cierto, recibieron descripciones $\mathrm{Cl}$. El único participante que consistentemente mantuvo porcentajes de acierto cercanos al $100 \%$ desde el entrenamiento hasta la prueba de transferencia extrarelacional (T3) fue el participante P9, quién no obtuvo ningún acierto en la siguiente prueba de transferencia (extradimensional, T4). Por último, ningún participante mostró una buena ejecución en T4, lo que descarta algún tipo de efecto de la retroalimentación o descripción de contingencias en dicha prueba de transferencia.

\section{Discusión}

Tal como lo afirmaron Ortiz et al. (2006) las descripciones de contingencias con más alto grado de precisión, la Específicas-Pertinentes, redujeron el número de sesiones necesarias para la adquisición de la conducta de discriminación condicional en los participantes que las recibieron, pues sólo necesitaron tres sesiones para cumplir con el criterio de logro para pasar de la fase de entrenamiento a la posprueba. Un efecto similar se encontró en algunos de los participantes que recibieron descripciones $\mathrm{Cl}$, pues también obtuvieron un porcentaje de respuestas correctas cercano al $100 \%$ durante tres sesiones consecutivas en el entrenamiento. Sin embargo la precisión de las descripciones EP promovió casi el 100\% de respuestas correctas en casi todos los participantes que las recibieron (con excepción de P3), mientras que tres de los participantes que recibieron descripciones $\mathrm{Cl}$ (P10, P11 y P14) tuvieron que enfrentar cuatro sesiones de entrenamiento por obtener porcentajes de respuestas correctas inferiores al $80 \%$ en una o dos sesiones. Una posible explicación de la falta de efectividad de las descripciones $\mathrm{Cl}$ puede deberse a que éstas se enfocaron en la presentación de ejemplos basados en las instancias, sin mencionar que los ejemplos hacían referencia al aprendizaje del criterio de relación que operaba en la tarea experimental (semejanza). En este sentido González-Becerra y Ortiz (2014) realizaron una investigación en la que los ejemplos de las descripciones $\mathrm{Cl}$ hacían referencia a las instancias, modalidades y relaciones. Los resultados mostraron que los participantes que recibieron descripciones $\mathrm{Cl}$ relacionales tenían un mejor desempeño que los 
participantes que recibieron descripciones $\mathrm{Cl}$ instanciales, tal como ha ocurrido en otras investigaciones en las que se ha manipulado la señalización de las propiedades instanciales, modales y relacionales de estímulos y respuestas (e,g Ribes, Moreno, \& Martínez, 1995; Ribes, Moreno, \& Martínez, 1998; Serrano, García, \& López, 2006; Serrano, García, \& López, 2008; Villanueva, Mateos, \& Flores, 2008).

Otro aspecto relevante respecto a los resultados en el Experimento 1 es que la presencia o ausencia de retroalimentación no tuvo un papel determinante en los resultados, ya que se encontró que la adquisición y transferencia de la conducta de discriminación condicional varió con relación a las descripciones de contingencias que recibieron los participantes y no con relación a la retroalimentación. Al parecer cuando las descripciones de contingencias corresponden a las condiciones de la tarea enfrentada por los participantes la retroalimentación adquiere un papel secundario (e. g., Ortiz, 2010; Ortiz \& González, 2010; Ortiz, González, Rosas, \& Alcaraz, 2006), pero cuando no hay correspondencia entre lo descrito y lo enfrentado en la tarea la retroalimentación puede adquirir mayor influencia en la respuesta que las descripciones de contingencias (e. g., Hayes, Brownstein, Zettle, Rosenfarb, \& Korn, 1986; Martínez, Ortiz, \& Rosas, 2007; Ortiz, de la Rosa, Padilla, Pulido, \& Vélez, 2008; Ribes \& Martínez, 1990).

Pese a que el efecto de las descripciones de contingencia se observó de manera clara en el entrenamiento, no fue así en las pruebas de transferencia, ya que se encontró que varios participantes que obtuvieron porcentajes de acierto cercanos al $100 \%$ desde el entrenamiento hasta la prueba de transferencia T1 no mantuvieron el mismo porcentaje de respuestas correctas en las siguientes pruebas de transferencia. Sólo un participante obtuvo casi el 100\% de respuestas correctas en la prueba de transferencia extrarelacional (T3), después de obtener un porcentaje de respuestas similar en sesiones precedentes y, además, todos los participantes obtuvieron porcentajes de respuestas correctas cercanos a cero en la prueba de transferencia extrdimensional (T4). Por lo tanto, siendo coherentes con el planteamiento teórico que se hizo al inicio de la investigación (e. g., Ribes \& López, 1985; Ribes, 1990a), los datos de la presente investigación parecen demostrar que la descripción de contingencias $\mathrm{EP} \mathrm{y} \mathrm{CI}$ no promueven el comportamiento sustitutivo referencial y no referencial. En cambio se esperaba que las descripciones $\mathrm{Cl}$ promovieran que la conducta de discriminación condicional aprendida en la situación geométrica del entrenamiento se transfiriera a la situación aritmética de la prueba de transferencia T4, tal como ocurrió en la investigación de Serrano, García y López (2009) para los participantes que recibieron retroalimentación de sus respuestas correctas e incorrectas en una TIM. Esto parece mostrar que la exposición indirecta a las contingencias mediante las descripciones $\mathrm{Cl}$ no tiene correspondencia con la exposición directa a las contingencias (e. g., Buskit \& Miller, 1986; Galizo, 1979; Hayes, Brownstein, Zettle, Rosenfarb, \& Korn, 1986; 
Ortiz, de la Rosa, Padilla, Pulido, \& Vélez, 2008; Shimoff, \& Catania, 1998). Sin embargo, el hecho de que la respuesta de discriminación condicional aprendida en el entrenamiento no se haya mantenido hasta la posprueba y las pruebas T1 pudo haber impedido evaluar el efecto de las descripciones sobre la prueba T4.

Uno de los resultados que más destaca de la presente investigación es la reducción en el porcentaje de respuestas correctas que algunos participantes mostraron al pasar del entrenamiento a la posprueba (P3) y/o de la posprueba a la prueba de transferencia extrainstancial (P6, P13 y P16). Básicamente la tarea del participante en la posprueba y en prueba de transferencia extrainstancial es responder a los estímulos de la tarea con base en el criterio de relación que aprendió en el entrenamiento. Esto no debería representar algún problema porque los estímulos utilizados en la posprueba son exactamente los mismos que los utilizados en el entrenamiento, mientras que en la prueba extrainstancial se siguen relacionando las mismas propiedades de los estímulos (color y forma) ante instancias de la misma categoría (figuras geométricas) que aquellas que se utilizan en el entrenamiento y en la posprueba. Sin embargo, revisando los resultados de otras investigaciones en las que se ha utilizado la TIM de primer orden (TIM-1) para el entrenamiento de una conducta de discriminación condicional también se encontró que al menos dos de sus participantes dejaban de obtener casi el $100 \%$ de las respuestas correctas cuando pasaba de la sesión de entrenamiento a la posprueba y/o a pruebas de transferencia extrainstancial (e. g., Guerrero-Radillo, \& Ortiz, 2007; Guerrero-Radillo \& Ortiz, 2009; Ortiz \& Cruz-Alaniz, 2011; Ortiz \& González, 2010). Cabe señalar que el efecto mencionado no se encuentra en investigaciones en las que se utiliza la TIM de segundo orden (TIM-2) para el entrenamiento de la conducta de discriminación condicional, pues en esos casos todos los participantes mantienen el alto porcentaje de respuestas correctas cuando pasan del entrenamiento a la posprueba o a una prueba de transferencia de baja complejidad (i.e., extrainstancial) (e. g., Serrano, García, \& López, 2006; Serrano, García, \& López, 2008; Serrano, García, \& López, 2009). La diferencia entre la TIM-1 y la TIM-2 es que la segunda presenta un estímulo de segundo orden que específica el criterio de relación entre el EM y uno de los ECós en cada sesión. En cambio en la primera tarea la especificación del criterio de relación depende de la retroalimentación y/o de la descripción de contingencias, variables que desaparecen en las sesiones de posprueba y pruebas de transferencia.

\section{Experimento 2}

La reducción en el porcentaje de respuestas correctas cuando se pasa del entrenamiento de una conducta de discriminación condicional a la posprueba o pruebas de transferencia en una TIM-1 es un efecto que ha pasado desapercibido por la baja 
cantidad de casos encontrados en algunas investigaciones (e. g., Guerrero-Radillo \& Ortiz, 2007; Guerrero-Radillo \& Ortiz, 2009; Ortiz \& Cruz-Alaniz, 2011; Ortiz \& González, 2010). También, se ha pasado por alto que quizás dicho efecto no sólo impide el mantenimiento de la conducta aprendida en el entrenamiento en sesiones posteriores, sino que además puede impedir la evaluación de las variables que se manipulan cuando se utiliza una TIM-1. En ese sentido se considera necesario realizar una réplica del Experimento 1 en la que se agreguen avisos de la sesión que enfrenta el participante en una TIM-1, bajo el supuesto de que los avisos especificarán el tipo de comportamiento esperado de manera análoga a la forma en la que lo hacen los estímulos de segundo orden en TIM-2. Si la hipótesis es correcta el Experimento 2 permitirá la evaluación de los efectos de las descripciones de contingencias sobre la adquisición y transferencia de una conducta de discriminación condicional.

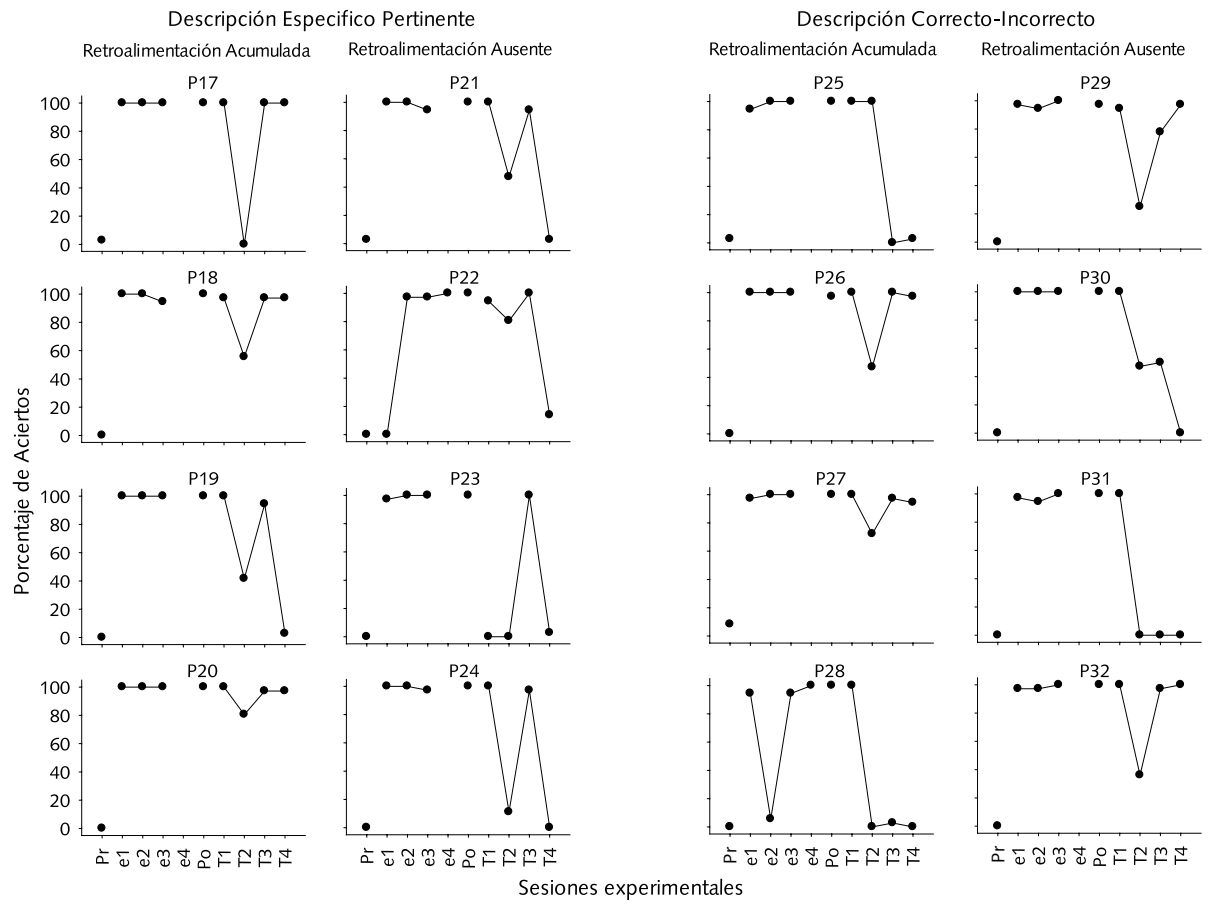

Figura 4. Porcentajes de aciertos obtenidos por los participantes del Experimento 2. Las abreviaciones representan sesiones experimentales y variables independientes, a saber: $\mathrm{Pr}=$ preprueba, $\mathrm{e}=$ entrenamiento, $\mathrm{Po}=$ posprueba, $\mathrm{T} 1=$ prueba de transferencia extrainstancial, $\mathrm{T} 2$ = prueba de transferencia extra modal, $\mathrm{T} 3=$ prueba de transferencia extrarelacional y $\mathrm{T} 4=$ prueba de transferencia extradimensional. 


\section{Método}

Participantes. Participaron 32 estudiantes universitarios con edades entre 18 y 25 años, sin experiencia en la tarea experimental, los cuales se asignaron al azar a los grupos experimentales. El contacto con los participantes fue a través de sus profesores, quienes les dieron puntos extras en su asignatura por la participación en el experimento.

Aparatos y materiales. Los mismos que se utilizaron en el Experimento 1.

Diseño. El mismo que se utilizó en el Experimento 1, sólo que en este caso los grupos que se conformaron por las variables manipuladas se denominaron Grupo 5 (EPacumulada), Grupo 6 (EP-Ausente), Grupo 7 (EP-Acumulada) y Grupo 8 (EP-Ausente).

Tarea experimental. La misma que se utilizó en el Experimento 1.

Procedimiento. Se utilizó casi el mismo procedimiento que en el Experimento 1, sólo se agregó al inicio de cada sesión una leyenda que indicaba la sesión experimental que estaba enfrentando el participante. El aviso se presentó en la misma diapositiva en la que se encontraban las descripciones, en la parte superior de la pantalla. En la sesión de preprueba apareció el aviso, "PREPRUEBA"; en las sesiones de entrenamiento, "ENTRENAMIENTO"; en la sesión de posprueba, "POSPRUEBA"; y en las sesiones de las pruebas de transferencia se presentaba el aviso con un número dependiendo de la prueba enfrentada, "PRUEBA DE TRANSFERENCIA X".

\section{Resultados}

En la Figura 3 se muestra el porcentaje de aciertos de los participantes del Experimento 2, las cuales fueron graficadas y organizadas de la misma manera que en la Figura 2. En cuanto a la ejecución de los participantes en el entrenamiento se encontró que todos obtuvieron porcentajes de aciertos cercanos al 100\% en la última sesión de dicha fase. Adicionalmente se encontró que casi todos los participantes requirieron de tres sesiones de entrenamiento y sólo dos participantes necesitaron de una sesión más (P22 y P28), resaltando que uno recibió descripciones EP y el otro CI. Por otra parte, la ejecución en la posprueba de todos los participantes fue cercana al 100\% de aciertos. Con relación a la ejecución en T1 sólo un participante (P23) no mantuvo los altos porcentajes de acierto mostrados en la posprueba. Los datos relacionados a la ejecución de los participantes en la posprueba y la prueba T1 denotan que la conducta aprendida en el entrenamiento se mantuvo hasta dichas pruebas, lo cual no ocurrió en el Experimento 1. Las ejecuciones en T2 decayeron en cuatro participantes (P17, P24, P28 y P31) que obtuvieron porcentajes de aciertos cercanos al $0 \%$ y siete participantes (P18, P19, P21, P26, P29, P30 y P32) que obtuvieron porcentajes cercanos al 50\%. Pero en la sesión T3 se encontró que algunos de los participantes que habían 
tenido un mal desempeño en la sesión T2 (P17, P18, P19, P20, P21, P22, P24, P26, P27, P29 y P32) mejoraron su ejecución obteniendo porcentajes de acierto cercanos al $100 \%$. Por último, se encontró que algunos de los participantes que habían mostrado un buen desempeño en la sesión T3, también lo hicieron en la sesión T4, de los cuales tres recibieron descripciones EP (P17, P18 y P20) y cuatro descripciones $\mathrm{Cl}$ (P26, P27, P29 y P32). De manera general las mejores ejecuciones en las pruebas de transferencia la obtuvieron los participantes que recibieron retroalimentación, con base en los resultados obtenidos por tres de los participantes (P17, P18 y P20) del Grupo 5 (EP-Acumulada) y por dos de los participantes (P26 y P27) del Grupo 7 (ClAcumulada).

\section{Discusión}

Uno de los resultados que más destacan del Experimento 2 en comparación al Experimento 1 es el mantenimiento de la conducta aprendida en el entrenamiento hasta la prueba T1 por la presencia de los avisos de la sesión enfrentada. La manipulación de dicha variable permitió evaluar los efectos de las variables de interés en ésta investigación (descripción y retroalimentación) porque permitió reconocer el tipo de conducta que se esperaba de los participantes en las sesiones que enfrentaban, algo que no ocurrió con los participantes del Experimento 1.

Con relación a los resultados obtenidos en el entrenamiento se encontró que todos los participantes obtuvieron altos porcentajes de aciertos en dicha fase experimental, independientemente del tipo de descripción recibida. Al parecer las descripciones $\mathrm{Cl}$ compensan la precisión de las descripciones EP mediante los ejemplos de conductas correctas e incorrectas, pues aunque las descripciones son morfológicamente diferentes, ambas propician la abstracción del criterio de relación (Ribes, 2000). Además, de manera similar a lo que ocurrió en el Experimento 1 y en otras investigaciones (e. g., Ortiz \& González, 2010) la retroalimentación no tuvo un efecto claro en el entrenamiento, siendo más relevante la descripción recibida. Sin embargo en la literatura se encuentran investigaciones en las que se manipuló el tipo de descripción y la densidad de retroalimentación y se encontraron resultados diferenciales en el entrenamiento de acuerdo al tipo de retroalimentación recibida (e. g., Cruz \& Ortiz, 2010; GuerreroRadillo \& Ortiz, 2007).

Uno de los datos más esperados en la presente investigación era la observación de altos porcentajes de acierto en la prueba de transferencia extrarelacional (T3) y extradimensional (T4) por su vinculación con el comportamiento extrasituacional y transituacional, respectivamente (Ribes, 1990b). Pero antes de continuar con el análisis es necesario aclarar que en la presente investigación se plantea una disyuntiva respecto a la propuesta original en la que se relacionan esas pruebas de transferencia con 
el comportamiento sustitutivo referencial y sustitutivo no referencial. A continuación se presentan las diferencias:

1) Se plantea que la prueba de transferencia extradimensional no promueve el comportamiento sustitutivo no referencial y, en consecuencia, no permite evaluar el ajuste coherente del participante al interrelacionar dos situaciones estableciendo los criterios lingüísticos que las vinculan para crear una nueva situación interactiva (Ribes, 2004; Ribes, 2007). Un par de casos que se relaciona al comportamiento transituacional (sustitutivo no referencial) son los del libro de la Rebelión de la Granja de George Orwell y la composición musical de "Las cuatro estaciones" de Vivaldi. En ambos casos los autores interrelacionaron dos situaciones de manera coherente para crear una nueva situación interactiva; en el primer caso una fábula relacionada a la corrupción del socialismo ruso por Stalin y el segundo caso cuatro conciertos relacionados a cada una de las estaciones del año.

2) Se rechaza el supuesto de que la función sustitutiva referencial se identifica con la respuesta acertada en una prueba de transferencia extrarelacional (Ribes, 1990, p. 218), es decir, cuando el participante cambia su criterio de respuesta (i.e., de identidad a semejanza). Si este supuesto se tomará al pie de la letra no habría necesidad de hacer pruebas de transferencia cuando se utiliza una TIM-2 debido a que tradicionalmente en el entrenamiento se entrena al participante para que relacione los estímulos con base en dos o más criterios de respuesta relacionados a un cambio en los estímulos de segundo orden (e. g., Serrano, García, \& López, 2006).

3) En vez de considerar a la prueba de transferencia extradimensional como una condición experimental que promueve el comportamiento transituacional, así como su evaluación, se propone que dicha prueba se relacione con la promoción y evaluación del comportamiento extrasituacional. La razón que justifica esta propuesta es que la prueba de transferencia extradimensional representa más bien un cambio de situación que la creación de una situación. Cuando el participante responde correctamente en dicha prueba debe de responder a "las mismas propiedades de los estímulos" (i.e., semejanza, diferencia), pero con estímulos de una dimensión distinta a aquellos estímulos con los que interactuó en el entrenamiento (i.e., de una dimensión geométrica a una aritmética). De tal manera que la interacción cumple con los requisitos que una función sustitutiva referencial tiene que involucrar (Ribes \& López, 1985; Ribes, 2004; Ribes, 2007): dos situaciones de interacción (i.e., dimensión geométrica y dimensión aritmética), dos momentos de respuesta (i.e., entrenamiento y transferencia), respuesta a las propiedades convencionales de los estímulos (i.e., propiedades geométricas / propiedades aritméticas) y congruencia en la 
respuesta del participante cuando la situación cambia (i.e., dejar de responder a la "semejanza"/"diferencia"/"identidad" de las propiedades geométricas de los estímulos para responder a la "semejanza"/"diferencia"/"identidad" de las propiedades aritméticas de los estímulos).

Volviendo a la discusión de los resultados, se encontró que tres participantes (P17, P18 y P19) que recibieron retroalimentación y descripciones $\mathrm{Cl}$ obtuvieron un porcentaje de respuestas correctas cercano al $100 \%$ en la prueba de transferencia extradimensional, mientras que sólo dos de los que recibieron descripciones $\mathrm{Cl}$ y retroalimentación (P26 y P27) tuvieron una ejecución similar en dicha prueba de transferencia. Sin embargo, si se analizan los altos porcentajes de aciertos obtenidos en la prueba T4, sin tomar en cuenta si los participantes recibieron retroalimentación o no, se encuentra que los que recibieron descripciones $\mathrm{Cl}$ superan en número a los que recibieron descripciones EP (3 a 4; P17, P18 y P19 vs. P26, P27, P28 y P30). Aunque hay ligeras diferencias en el número de participantes que en cada grupo experimental logró obtener altos porcentajes de aciertos en la prueba T4, el dato relevante es que ambos tipos de descripciones promueven lo que en esta investigación se considera comportamiento sustitutivo referencial.

En el caso de los participantes que recibieron descripciones $\mathrm{Cl}$, la generalización se favoreció por la descripción de respuestas correctas e incorrectas, tal como lo asumían Engelmann y Carnine (1991). Sin embargo, parecería extraño encontrar que los participantes que recibieron descripciones EP hayan generalizado la conducta aprendida, cuando no se expusieron a las contingencias irrelevantes de la tarea, ya que estos no se expusieron a las contingencias irrelevantes de la tarea directa (por la retroalimentación) o indirectamente (por descripciones). Pero, es necesario tener en cuenta que en las descripciones EP se hizo referencia a las propiedades modales (color o forma) y relacionales (semejanza) de los estímulos a los que respondieron en el entrenamiento y que dicho tipo de descripción promueve altos porcentajes de acierto en las pruebas de transferencia (e. g., González-Becerra \& Ortiz, en revisión; Ribes, Moreno, \& Martínez, 1995; Ribes, Moreno, \& Martínez, 1998; Serrano, García, \& López, 2006; Serrano, García, \& López, 2008; Villanueva, Mateos, \& Flores, 2008).

\section{Discusión General}

Afortunadamente los avisos que se presentaron a los participantes del Experimento 2 para especificar que sesión estaban enfrentando permitieron evaluar el efecto de las variables retroalimentación y descripción en la adquisición y transferencia de una conducta de discriminación condicional en una TIM-1. Los avisos se agregaron a la TIM-1 bajo el supuesto de que dicha tarea carece de señales que especifiquen el tipo de comportamiento esperado por el participante en las sesiones en las que no 
recibe descripciones de contingencias ni retroalimentación (i.e., posprueba, pruebas de transferencia). Para Ortiz y González (2010) la falta del aviso pudo haber influido en que algunos participantes se autoinstruyeran (e. g., Cerutti, 1989) en cambiar de criterio de relación porque la sesión posprueba era distinta de la del entrenamiento. En cambio los participantes que aprenden una respuesta de discriminación condicional en una TIM-2 no tienen problemas para mantener la conducta aprendida en el entrenamiento en la posprueba y/o en las pruebas de transferencia porque el estímulo de segundo orden específica, en cada ensayo, el tipo de criterio de relación vigente en la interacción (e. g., Ribes, Moreno, \& Martínez, 1995; Serrano, García, \& López, 2006; Serrano, García, \& López, 2008; Serrano, García, \& López, 2010). Un ejemplo de los efectos que se mencionaron para la TIM-1 y la TIM-2 se encuentra en la investigación de Serrano et al. (2009), en la que se realizaron dos experimentos con cada tarea experimental para evaluar los efectos de la retroalimentación sobre una conducta de discriminación condicional. Los autores expusieron a los participantes a una preprueba, una sesión de entrenamiento, tres pruebas de transferencia (similares a T1, T2 y T4) y una posprueba (en esa secuencia), en las que tenían que responder a tres criterios de relación (semejante, idéntico y diferente). Para los participantes que interactuaban en una TIM-1 se presentaban pantallas entre los ensayos del entrenamiento y las pruebas de transferencia que avisaban del cambio de relación, lo cual no se hizo en la preprueba y posprueba. Por otra parte los participantes expuestos a la TIM-2 tuvieron en todas las sesiones experimentales estímulos de segundo orden que especificaban el cambio de criterio de relación. Los resultados mostraron que los participantes que habían tenido altos porcentajes de aciertos en el entrenamiento y pruebas de transferencia en una TIM-2 mantuvieron su ejecución hasta la posprueba (la última sesión del experimento). En cambio los participantes que habían logrado altos porcentajes de aciertos en el entrenamiento y pruebas de transferencia en una TIM-1 obtuvieron porcentajes de aciertos cercanos a cero en la posprueba. Quizás en esta investigación también la falta de un aviso que especificara el tipo de criterio de relación que se esperaba en dicha prueba provocó el cambio de criterio de relación. Por lo tanto, aunque ambos tipos de tareas permiten el estudio de los efectos de la retroalimentación y las descripciones de contingencias es más recomendable utilizar la TIM-2 porque no requiere de avisos que especifiquen el tipo de comportamiento que se espera del participante en cada una de las sesiones de experimentales.

Por otra parte, es de destacar que pese a que las propiedades de los estímulos y las respuestas que se señalan en las descripciones de contingencias EP y $\mathrm{Cl}$ son muy distintas, pues la primeras señalan propiedades instanciales, modales y relacionales mientras que las segundas sólo señalan propiedades instanciales, ambas favorecieron el aprendizaje y transferencia de la conducta de discriminación condicional. Una posible explicación es que las descripciones EP que se utilizaron en la presente inves- 
tigación hacen referencia a las propiedades modales y relacionales de los estímulos, propiedades que parecen favorecer la adquisición y transferencia de una conducta de discriminación condicional (Serrano, García, \& López, 2006; Serrano, García, \& López, 2008; Serrano, García, \& López, 2010; Villanueva, Flores, \& Mateos, 2008). Por otra parte, pese a que las descripciones $\mathrm{Cl}$ que recibieron los participantes del Experimento 2 sólo describían las propiedades instanciales de los estímulos y las respuestas, estas promueven, mediante ejemplos de respuestas correctas e incorrectas, altos porcentajes de respuestas correctas. En este sentido, ambos tipos de descripciones de contingencias permiten el reconocimiento del criterio de igualación, sólo que las descripciones EP lo hacen de manera explícita y las descripciones $\mathrm{Cl}$ de manera implícita.

Es necesario señalar que los altos porcentajes de aciertos que lograron algunos de los participantes del Experimento 2 en la prueba de transferencia extradimensional (t4) se han interpretado tradicionalmente como comportamiento transituacional (e. g., Carpio, Pacheco, Carranza, Flores, \& Canales, 2003; Irigoyen, Carpio, Jiménez, Silva, \& Acuña, 2002; Ribes, 1990; Serrano, García, \& López, 2006; Serrano, García, \& López, 2008; Serrano, García, \& López, 2010; Villanueva, Flores, \& Mateos, 2008). Sin embargo, como se comentó anteriormente, en la presente investigación se propone que el comportamiento acertado en las pruebas de transferencia extradimensional corresponde más bien al comportamiento extrasituacional. El argumento es que los participantes transfieren el criterio de relación aprendido en una situación geométrica, durante el entrenamiento, a una situación aritmética, en la prueba de transferencia T4. Se asume que ambas situaciones son distintas porque "la semejanza", "identidad" o "diferencia" a la que se responde en una situación geométrica esta interrelacionada a las propiedades físicoquímicas de los estímulos (i.e., color, forma, tamaño), en cambio, en la situación aritmética "la semejanza", "identidad" o "diferencia" ya no tiene relación con la morfología de los estímulos, sino con las propiedades que se les atribuye en la enseñanza de las matemáticas. De tal manera que el participante desliga su respuesta de las propiedades morfológicas de los estímulos para responder, congruentemente, a "las propiedades aritméticas" de los estímulos que se presentan en la prueba de transferencia extradimensional (Ribes, 2004, 2007).

Otro aspecto que no se ha discutido de las investigaciones dedicadas al estudio de la intra, extra y transituacionalidad de la respuesta de discriminación condicional es el sesgo que implica "entrenar" y "transferir" una conducta aprendida por el participante previo a su exposición a la TIM, a saber: la respuesta a la semejanza, diferencia e identidad entre estímulos con propiedades geométricas y aritméticas. Si bien, uno de los criterios de inclusión a este tipo de experimentos es que el participante carezca de experiencia con la TIM, nunca se evalúa que este pueda o no pueda relacionar figuras geométricas y/o números por semejanza, identidad o diferencia previo a su 
participación en el experimento. De hecho, por la escolaridad de los participantes de la mayoría de los estudios (que por lo general son de primaria o licenciatura) se podría asumir que estos ya son capaces de relacionar figuras geométricas y números por semejanza, identidad o diferencia. Entonces, ¿bajo qué argumento se dice que se "entrena" y "transfiere" una conducta discriminación condicional con un(os) criterio(s) de relación que ya había(n) sido "aprendido(s)" y "transferido(s)" en circunstancias distintas a las del experimento? Nuestra respuesta particular es que no hay argumentos $y$, adicionalmente, queremos aclarar que no advertimos esta contrariedad hasta que terminamos la presente investigación, pues utilizamos los criterios de relación que "tradicionalmente se entrenan y transfieren" sin pensar en sus implicaciones. En este sentido se asume que el comportamiento sustitutivo referencial que se relacionó a las respuestas acertadas de los participantes en la prueba de transferencia extradimensional ya había sido aprendido por el participante antes del experimento, de tal forma que la investigación sólo demuestra que el participante también puede responder en ese nivel de complejidad en una TIM. Quizás por esto Peña-Correal, Ordóñez, Fonseca y Fonseca (2012) encontraron en el conjunto de estudios que analizaron que la manipulación de descripciones de contingencia y retroalimentación sólo son facilitadoras y no posibilitadoras del comportamiento sustitutivo referencial en TIM.

Derivado lo que aquí se discutió se propone la realización de investigaciones en las que se entrene en una TIM un comportamiento "novedoso" para los participantes con el objetivo de evaluar el desarrollo del comportamiento sustitutivo referencial y no su actualización. Tal vez así se pueda evaluar si las cualidades de las descripciones de contingencias o la retroalimentación son facilitadores o posibilitadoras del comportamiento sustitutivo referencial.

\section{Referencias}

Buskist, W. F. \& Miller, H. L. (1986). Interaction between rule and contingencies in the control of human fixed-interval performance. The Psychological Record, 36, 109-116.

Carpio, C., Pacheco, V., Carranza, N., Flores, C. \& Canales, C. (2003). Tipos de retroalimentación en el aprendizaje de términos metodológicos de la psicología experimental. Anales de Psicología, 19, 97-105.

Cerutti, D. T. (1989). Discrimination theory of rule-governed behavior. Journal of the Experimental Analysis of Behavior, 51, 259-276.

Cumming, W.W. \& Berryman, R. (1961). Some data on matching behavior in the pigeon. Journal of the Experimental Analysis of Behavior, 4(3), 281-284.

Cruz, Y., \& Ortiz, G. (2011). El papel de la precisión instruccional y la retroalimentación en la ejecución y descripciones poscontacto. Revista Mexicana de Análisis de 
la Conducta, 37(1), 69-87.

Engelmann, S., \& Carnine, D. (1991). Theory of instruction: principles and applications. Eugene, OR: ADI Press.

Galizio, M. (1979). Contingency-shaped and rule-governed behavior: instructional control of human loss avoidance. Journal of the Experimental Analysis of Behavior, 31, 53-70.

González-Becerra, V., \& Ortiz, G. (2014). Efectos del tipo y contenido de descripciones pre-contacto contingencial sobre la conducta de discriminación condicional y descripciones post-contacto. Acta Colombiana de Psicología, 17(1), 11-23.

Guerrero-Radillo, A., \& Ortiz, G. (2007). El papel de la retroalimentación y la ausencia o presencia de instrucciones en la elaboración de descripciones en tareas de discriminación condicional. Acta Colombiana de Psicología, 10, 5-13.

Guerrero-Radillo, A. P., \& Ortiz, G. (2009). Efectos de un preentrenamiento verbal sobre descripciones poscontacto y ejecución instrumental. IPyE: Psicología y Educación, 3, 1-19.

Hayes, S.C., Brownstein, A.J., Zettle, R.D., Rosenfarb, I., \& Korn, Z. (1986). Rule-governed behavior and sensitivity to changing consequences of responding. Journal of the Experimental Analysis of Behavior, 45, 237-256.

Martínez, H., Ortiz, G. \& González, A. (2007). Efectos diferenciales de instrucciones y consecuencias en ejecuciones de discriminación condicional humana. Psicothema, 19(1), 14-22.

Moreno, D., Ribes, E. \& Martínez, H. (1994). Evaluación experimental de la interacción entre el tipo de pruebas de transferencia y la retroalimentación en una tarea de discriminación condicional bajo aprendizaje observacional. Revista Latina de Pensamiento y Lenguaje, 2, 245-286.

Ortiz, G. (2010). Precisión de descripciones y conocimiento de la finalidad de la descripción poscontacto sobre la ejecución, elaboración y transmisión de descripciones. Acta Comportamentalia, 18(2), 189-213.

Ortiz, G., \& Cruz-Alaniz, Y. (2011). El papel de la precisión instruccional y la retroalimentación en la ejecución y descripciones poscontacto. Revista Mexicana de Análisis de la Conducta, 31(1), 69-87.

Ortiz, G., \& González, V. (2010). Efecto de dos tipos de descripciones precontacto sobre la ejecución instrumental y descripciones poscontacto en tareas de igualación de la muestra. Acta Colombiana de Psicología, 13, 115-126.

Ortiz, G., González, A., \& Rosas, M. (2008). Una taxonomía para el análisis de descripciones pre y post contacto con arreglos contingenciales. Acta Colombiana de Psicología, 11(1), 45-53.

Ortiz, G., de la Rosa, E., Padilla, R., Pulido, E. \& Vélez, H. (2008). Efecto de la precisión e historia instruccional en la insensibilidad a las consecuencias en tareas de 
igualación a la muestra de primer orden en humanos. Acta Comportamentalia, 16(2), 167-181.

Ortiz, G., González, A., Rosas, M., \& Alcaraz, F. (2006). Efectos de la precisión instruccional y la densidad de retroalimentación sobre el seguimiento instruccional, la elaboración y transmisión de descripciones en tareas de discriminación condicional. Acta Comportamentalia, 14, 103-130.

Pérez, A. \& Flores, C. (2007). Papel de la retroalimentación en la adquisición y transferencia de discriminaciones condicionales en estudiantes universitarios. Enseñanza e Investigación en Psicología, 12(1), 65-77.

Peña-Correal, T., Ordóñez, S., Fonseca, J., \& Fonseca, C. (2012). La investigación empírica de la función sustitutiva referencial. En R. Pérez-Almonacid \& M. Padilla (Eds.), La función Sustitutiva Referencial: análisis histórico-crítico/ avances y perspectivas (pp. 35-100). USA: University Press of the South.

Ribes, E. (1990a). Psicología y Salud. México: Trillas.

Ribes, E. (1990b). Psicología General. México: Trillas.

Ribes, E. (1992). Sobre el tiempo y espacio psicológicos. Acta Comportamentalia, 10, 71-84.

Ribes, E. (2004). Acerca de las funciones psicológicas: un post-scriptum. Acta Comportamentalia, 12(2), 117-127.

Ribes, E. (2006). Conceptos, categorías y conducta: reflexiones teóricas. Revista Latina de Pensamiento y Lenguaje, 15, 5-23.

Ribes, E. (2007). Estados límites de campo, medios de contacto y análisis molar del comportamiento: reflexiones teóricas. Acta Comportamentalia, 15(2), 229-259.

Ribes, E. (2012). Las funciones sustitutivas de contingencias. En: M. Padilla y R. PérezAlmonacid (Eds.), La función sustitutiva referencial: análisis histórico crítico / avances y perspectivas, (p. 14-34). United States: University Press of the South.

Ribes, E., Ontiveros, C., Torres, C., Calderón, G, Carvajal, J., Martínez, C., \& Vargas, I. (2005). La igualación de la muestra como selección de los estímulos de segundo orden: efectos de dos procedimientos. Revista Mexicana de Análisis de la Conducta, 31, 1-22.

Ribes, E., Moreno, D., \& Martínez, C. (1998). Second-order discrimination in humans: the roles of explicit instructions and constructed verbal responding. Behavioral Processes, 42, 1-18.

Ribes, E., Moreno, D. \& Martínez, C. (1995). Efecto de distintos criterios verbales de igualación en la adquisición y transferencia de una discriminación condicional de segundo orden con humanos. Acta Comportamentalia, 3(1), 27-54.

Ribes, E. \& López, F. (1985). Teoría de la conducta: un análisis de campo y paramétrico. México: Trillas.

Serrano, M., García, G., \& López, A. (2010). Textos descriptivos de contingencia y 
retroalimentación en igualación de la muestra por humanos. International Journal of Psychology and Psychological Therapy, 10(1), 177-187.

Serrano, M., García, G., \& López, A. (2009). Efectos de la retroalimentación para las respuestas de igualación correctas o incorrectas en la adquisición y transferencia de discriminaciones condicionales. Revista Mexicana de Análisis de la Conducta, 35(1), 113-134.

Serrano, M., García, G., \& López, A. (2008). Textos descriptivos de contingencia como instrucciones iníciales en tareas de igualación de la muestra. Acta Comportamentalia, 16(2), 333-346.

Serrano, M., García, G., \& López, A. (2006). Textos descriptivos de contingencias como estímulos selectores en igualación de la muestra con humanos. Acta Comportamentalia, 14(2), 131-143.

Skinner, B.F. (1950). Are theories of learning necessary? Psychological Review, 57, 193-216.

Shimoff, E. \& Catania, A.C. (1998). The verbal governance of behavior. En K. A. Lattal, \& M. Perone (Eds.), Handbook of research Methods in human operant Behavior (pp. 371-404). USA: Plenum Press.

Torres, C., Ortiz, G., Rangel, N., \& González, V. (2010). Análisis del concepto de situación en la estructuración de las funciones psicológicas. En R. Pérez-Almonacid \& M. Padilla (Eds.), La función sustitutiva referencial: análisis histórico-crítico/ avances y perspectivas (pp. 149-167). USA: University Press of the South.

Varela, J., \& Quintana, C. (1995). Comportamiento inteligente y su transferencia. Revista Mexicana de Análisis de la Conducta, 21, 47-66.

Villanueva, S., Flores, C., \& Mateos, R. (2008). Efectos del contenido y distribución de la retroalimentación sobre la discriminación condicional de segundo orden. Acta Comportamentalia, 16(2), 211-221. 


\section{Apéndice A}

Tipos de descripciones de acuerdo a la sesión experimental enfrentada

Pre-prueba

A continuación en- - En seguida se te contraras algunas figuras, selecciona de las de abajo la que creas que va con la de arriba. la siguiente tarea. presentaran algunas instrucciones para que ejecutes correctamente

A continuación en la pantalla aparecerán cuatro figuras, una arriba y tres abajo. De las figuras de abajo una será diferente en color y en forma; otra semejante ya sea en color o en forma, pero no ambas al mismo tiempo; y otra idéntica en color y en forma respecto a la figura de arriba. Tu tarea consiste en elegir, de entre aquellas que pueden ser seleccionadas, la figura que tenga el mismo color o forma (pero no ambas al mismo tiempo).

Para llevar a cabo tu elección deberás de mover y oprimir el botón izquierdo del "mouse".

Al final de la sesión habrá una diapositiva en la que aparecerá en un recuadro el total de aciertos que obtuviste.
Para realizar la siguiente tarea necesitas tomar en cuenta los siguientes ejemplos de elección correcta e incorrecta:

\section{Elección correcta}

Cuando hay un triángulo rojo arriba la elección correcta es el triángulo verde.

Cuando hay un triángulo

amarillo arriba la elección correcta es el rectángulo amarillo.

Cuando hay un cuadrado rojo arriba la selección correcta es el cuadrado amarillo.

Cuando hay un cuadrado blanco arriba la selección correcta es el círculo blanco.

Cuando hay un círculo amarillo arriba la selección correcta es el círculo rojo. Cuando hay un círculo verde arriba la selección correcta es el triángulo verde.

Cuando hay un rectángulo blanco arriba la selección correcta es el rectángulo rojo.

Cuando hay un rectángulo rojo arriba la selección correcta es el triángulo rojo.
A continuación se evaluará lo que previamente has aprendido. No recibirás instrucciones ni información acerca de tu desempeño.
Pruebas de transferencia
A continuación se evaluará lo que previamente has aprendido. No recibirás instrucciones ni información acerca de tu desempeño. 
80

Pre-prueba

Entrenamiento Específico Pertinente

Entrenamiento
Correcto-Incorrecto

Post-prueba

Pruebas de transferencia

\section{Elección Incorrecta}

Cuando hay un cuadrado rojo arriba la elección incorrecta es el cuadrado rojo. Cuando hay un triángulo verde arriba la elección incorrecta es el triángulo verde. Cuando hay un rectángulo blanco arriba la elección incorrecta es el círculo amarillo.

Cuando hay un triángulo amarillo arriba la elección incorrecta es el cuadrado verde.

Cuando hay un rectángulo amarillo arriba la elección incorrecta es el rectángulo amarillo.

Cuando hay un triángulo blanco arriba la elección incorrecta es el triángulo blanco

Cuando hay un cuadrado verde arriba la elección incorrecta es el triángulo rojo. Cuando hay un triángulo rojo arriba la elección incorrecta es el rectángulo blanco. 